\title{
A assistência de enfermagem ao paciente submetido à artroplastia total de quadril e a importância dos cuidados no período pós operatório
}

\author{
The nursing care of patients undergoing total hip arthroplasty and the importance of care in \\ the postoperative period
}

\section{La atención de enfermería de los pacientes sometidos a una artroplastia toral de cadera y de la importancia del cuidado en el postoperatorio}

\begin{abstract}
Resumo: A Artroplastia Total de Quadril (ATQ) é uma cirurgia de reconstituição da articulação coxo femural pela substituição por prótese. A principal condição que leva à ATQ é a degeneração da cartilagem articular - os objetivos dessa cirurgia são alívio da dor, restauração e melhora da função articular. Destacar os cuidados de enfermagem específicos direcionados ao paciente submetido à ATQ no período pós operatório, bem como a importância da sistematização da assistência de enfermagem (SAE). Utilizou-se artigos das bases de dados SciELO e LILACS. A enfermagem tem papel fundamental no processo perioperatório dos pacientes submetidos à ATQ. A SAE é uma ferramenta que auxilia o enfermeiro a prestar um cuidado individualizado amplo, abrangendo orientações em todas as fases da internação e no domicilio, visando eliminar possibilidades de iatrogenias, proporcionar uma reabilitação adequada e capacitar o cuidador para alta hospitalar.
\end{abstract}

Descritores: Artroplastia Total de Quadril, Assistência de Enfermagem, Pós Operatório.

\begin{abstract}
A Total Hip Arthroplasty (THA) is a repair surgery for femoral hip joint replacement by prosthesis. The main condition that leads to ATQ is the degeneration of articular cartilage - the goals of this surgery are pain relief, restoration and improved joint function. Highlight the specific nursing care directed to the patient undergoing THA in the postoperative period as well as the importance of systematization of nursing care (SAE). We used items from the SciELO and LILACS. Being used as bibliographic references published in the last five years. Nursing plays a key role in the perioperative process of patients undergoing THA. The SAE is a tool that helps nurses to provide ample individualized care, including guidelines at all stages of admission and in the home, to eliminate possibilities iatrogenic diseases, provide adequate rehabilitation and empower the caregiver to hospital.
\end{abstract}

Descriptors: Total Hip Arthroplasty, Nursing Care, Postoperative.

Resumen: A Artroplastia Total de Cadera (THA) es una cirugía de reparación de la cadera femoral de reemplazo articular por una prótesis. La condición principal que conduce a ATQ es la degeneración del cartílago articular - los objetivos de esta cirugía son el alivio del dolor, la restauración y la mejora de la función articular. Resalte el cuidado de enfermería específica dirigida a la THA paciente sometido en el postoperatorio, así como la importancia de la sistematización de la asistencia de enfermería (SAE). Utilizamos elementos de la SciELO y LILACS. Enfermería tiene un papel clave en el proceso perioperatorio de los pacientes sometidos a una artroplastia. EI SAE es una herramienta que ayuda a las enfermeras para proporcionar un amplio cuidado individualizado, incluyendo directrices en todas las fases de admisión y en el hogar, para eliminar posibilidades de enfermedades iatrogénicas, proporcionar una rehabilitación adecuada y la autonomía de la cuidadora al hospital.

Descriptores: Artroplastia Total de Cadera, Cuidados de Enfermería, Post-operatorio.

Aline Barbosa Soares, André Moreno Silva, Gisele Duarte da Silva, I sabel Cristina Gomes Leite de Siqueira, Jéssica Pamponet Acadêmicas e Acadêmico do Curso de Enfermagem do Centro Universitário São Camilo.

Maysa Paloma da Cruz Acadêmica do Curso de Enfermagem do Centro Universitário São Camilo. E-mail: maysacruz@hotmail.com

Priscila Quiles Acadêmica do Curso de Enfermagem do Centro Universitário São Camilo. 


\section{I ntrodução}

A artroplastia total de quadril (ATQ) é uma cirurgia ortopédica, indicada para pacientes com doença degenerativa da cartilagem articular, representada pela osteoartrose do quadril, ou em pacientes vítimas de fratura do colo femoral ${ }^{1}$.

A osteoartrose pode ser primária (idiopática), ou secundária, quando a degeneração da cartilagem ocorre devido a alguma outra patologia que acometa o quadril, como a necrose asséptica da cabeça do fêmur, as doenças reumáticas, as displasias congênitas do quadril e as sequelas de trauma (fraturas do colo e cabeça do fêmur e acetabulares não identificadas). Por isso a maioria dos pacientes submetidos a este procedimento são idosos, apesar de não descartar pacientes adultos e jovens ${ }^{2}$.

A ATQ foi considerada a cirurgia do século inventada por John Chamley, ortopedista inglês, em 1966 e revolucionou o tratamento de patologias que acometem o quadril e a região coxofemural ${ }^{3}$.

A Artroplastia Total de Quadril é uma cirurgia limpa e eletiva de caráter reconstrutor, onde há substituição do quadril ou articulações lesionadas de maneira irreversível, a fim de minimizar a dor do paciente, que na maioria dos casos são idosos, pois na velhice há diminuição de massa óssea e desequilíbrio de cálcio e aumento de pessoas com osteoporose ${ }^{4}$.

Quanto à forma de fixação da prótese esta pode ser cimentada ou não cimentada (fixação por parafuso), ambas podem ter em sua composição metal/polietileno; cera/polietileno; metal/metal; cerâmica/cerâmica, isso dependerá da escolha do cirurgião, bem como o material das próteses ${ }^{3}$.

A organização mundial de saúde estima que $25 \%$ dos indivíduos acima de 65 anos sofrem de dor e incapacidade associados à osteoartrose, portanto a artroplastia total do quadril tornou-se uma intervenção cirúrgica frequente e cotidiana, principalmente devido à maior prevalência de osteoartrose 2 .

Conforme o Protocolo Gerenciado Artroplastia Total do Quadril do Hospital Israelita Albert Einstein - $\mathrm{HIAE}^{1}$, a principal indicação para a abordagem cirúrgica é o relato de dor de caráter progressivo e limitante, responsável pela redução da mobilidade articular, dificuldade de movimentação e deambulação em que não há melhora com o tratamento clínico.

O comprometimento da capacidade funcional do idoso tem implicações importantes para a família, para a comunidade, para o sistema de saúde e para a vida do próprio idoso, uma vez que a incapacidade ocasiona maior vulnerabilidade e dependência na velhice, contribuindo para a diminuição do bem-estar e da qualidade de vida dos idosos $^{5}$. Dessa forma, a incapacidade funcional é um problema social, que traz maior risco de institucionalização e altos custos para os serviços de saúde.

Para que haja um bom resultado na ATQ é necessário que a enfermagem, junto com a equipe multiprofissional, trabalhe com atenção e respeito, afim de que toda assistência prestada esteja isenta de complicações, havendo longevidade da prótese, garantindo ao paciente menos dor e capacidade produtiva.

\section{Objetivo}

Destacar os cuidados de enfermagem específicos direcionados ao paciente submetido à Artroplastia Total de Quadril (ATQ), enfatizando os cuidados no pós-operatório, as complicações cirúrgicas, prescrição de enfermagem e orientação para alta, utilizando a Sistematização da Assistência de Enfermagem (SAE).

\section{Material e Método}

Esta pesquisa caracteriza-se como de natureza descritiva, retrospectiva e de revisão bibliográfica, tendo como objeto os estudos publicados sobre o tema em questão dos últimos dez anos, em periódicos nacionais, indexados e especializados na área de saúde. A escolha em nível nacional foi com a intenção de conhecer os modelos de assistência aos pacientes submetidos à Artroscopia total de quadril através de estudos publicados nos países.

As bases de dados acessadas foram: SciELO e LILACS para o levantamento dos estudos científicos de 2005 à 2009, além do acervo de 2001 à 2009 da Biblioteca Pe. Inocente Radrizzani. Os critérios de inclusão foram: estudos publicados em periódicos nacionais que constam nas referidas bases de dados; textos acessados na íntegra, pesquisas originais e abordado os temas: Artroplastia Total de Quadril, assistência de enfermagem e os cuidados 
específicos aos pacientes submetidos à ATQ, Qualidade de vida no pós operatório de pacientes submetidos à esta cirurgia. Foram utilizados os seguintes descritores (BIREME - Biblioteca Regional de Medicina Descritores da Ciência de Saúde - DeCS) e recurso booleano and ou or. Para apresentação do plano de assistência foram utilizadas as taxonomias de North American Nursing Diagnosis Association (NANDA 20092011), Classificação das Intervenções de Enfermagem (NIC) e Classificação dos Resultados de Enfermagem (NOC).

\section{Resultados e Discussão}

\section{Período pré operatório}

O período pré operatório imediato compreende desde a véspera da cirurgia até o momento em que o paciente é recebido no centro cirúrgico, incluindo a visita pré-operatória de enfermagem. O enfermeiro do centro cirúrgico realiza a visita pré operatória atuando em três fases distintas: levantamento de problemas por meio do prontuário do paciente, utilizando dados da história, evolução clínica e de enfermagem, anotações de enfermagem e resultados de exames, com o objetivo de detectar as necessidades básicas afetadas. Com base no levantamento de problemas, o enfermeiro realiza a entrevista, prestando orientações ao paciente e familiares. Há esclarecimentos sobre o ato anestésico cirúrgico, havendo a elaboração do plano de assistência para o período transoperatório, prescrevendo para cada problema levantado uma assistência de enfermagem ${ }^{4}$.

Os pacientes e seus familiares devem ser orientados sobre o que devem esperar antes, durante e após a cirurgia. As explicações dadas pela enfermagem também devem ser comunicadas aos demais responsáveis pelo cuidado, por meio da documentação no registro do paciente. De acordo com Eliopoulos ${ }^{6}$ as preocupações, as perguntas e receios devem ser identificados pela enfermeira durante a investigação e a preparação pré-operatória, e o médico deve ter conhecimento sobre esses achados.

Deve-se revisar com o médico as medicações que o paciente está recebendo para determinar quais delas devem ser mantidas durante a hospitalização, checar com o paciente se o mesmo fez uso de antibióticos, tranquilizantes ou outros medicamentos antes de sua hospitalização, já que ocasionalmente eles esquecem ou relutam em passar esse tipo de informação ${ }^{6}$.

Como as funções cardíacas e respiratórias podem ser alteradas por determinadas drogas, é importante garantir que essa informação seja comunicada ao médico ${ }^{7}$.

É importante avaliar as funções cardiovascular, respiratória, renal e hepática. Avaliar o estado neurovascular no membro que irá sofrer a substituição articular, e seus resultados serão comparados com o pósoperatório a fim de identificar alterações e déficits ${ }^{8}$.

\section{Intraoperatório}

A fase intraoperatória compreende desde a entrada do paciente na sala de operação até sua ida para a sala de recuperação anestésica. Acredita-se que este é o momento mais estressante para o paciente e para a equipe. Algumas ações realizadas na sala de cirurgia devem corresponder não só às atividades técnicas, mas principalmente as expectativas do paciente, que deve ser respeitado em suas crenças e valores, bem como oferecer cuidado, atenção e segurança aos seus medos e suas necessidades. A equipe de enfermagem deve atentar-se também para garantir o bom funcionamento de todos os equipamentos utilizados durante a cirurgia, adotar precauções especiais na sala de cirurgia (vestimenta cirúrgica impermeável), observar o sistema de ar que deve estar limpo, a fim de diminuir o material particulado, a contagem de bactérias no ar e o risco de próteses infectadas ${ }^{4}$.

O enfermeiro deve realizar colocação de coxim para proteção do cliente, principalmente nas proeminências ósseas, preparar bisturi elétrico e placa de aterramento, assepsia da pele e delimitação do campo cirúrgico, além de transferir o paciente da maca para mesa cirúrgica com ajuda do anestesiologista e monitorar a presença de sonda vesical, acesso venoso e sinais vitais ${ }^{4}$.

A anestesia mais utilizada na Artroplastia Total de Quadril é a geral por inalação ou endovenosa, ou ainda bloqueio epidural. A intubação endotraqueal e o uso de ventilação mecânica são imprescindíveis para manter o sistema cardiopulmonar em bom funcionamento. A sondagem vesical é outro importante item que não pode ser esquecido, pois com a anestesia o paciente perde força 
muscular e de esfíncter. Porém o uso da sonda vesical é determinado pelo cirurgião responsável ${ }^{4}$.

É importante destacar a importância do uso de EPIS nessa cirurgia, que tem sangramento abundante, podendo até necessitar de uma reposição sanguínea para o paciente. Dentre os EPIs destaca-se a importância do uso de óculos, para proteger a mucosa ocular que tem grande chance de ser contaminada.

A parada cardíaca súbita logo após a cimentação e colocação do componente femoral pode ocorrer e tem etiologia específica, sendo que esta se deve à embolia gordurosa maciça. Êmbolos gordurosos são achados comuns na autópsia de pacientes que morreram durante Artroplastia Total do Quadril ${ }^{9}$. Além da falência cardíaca, pode ocorrer hipotensão transitória, presente em alguns indivíduos durante a inserção da prótese no canal femoral.

Manifestações cardiovasculares indesejáveis têm sido associadas à utilização do polimetilmetacrilato (PMMA). A compreensão da fisiopatologia desses eventos mostra que essas intercorrências são devidas à embolia de fragmentos tissulares, ao invés de resultar da intoxicação pelo PMMA. Esses fragmentos causam a liberação de substâncias vasoativas, que levam à vasodilatação e consequentemente a queda da pressão arterial e do débito cardíaco ${ }^{9}$.

\section{Sala de Recuperação Anestésica (SRPA)}

Este período é o pós operatório imediato, abrange desde a alta do paciente da sala de operação até a saída da sala de recuperação pós anestésica.

o enfermeiro deve observar esses pacientes continuamente, elaborando a prescrição e evolução de enfermagem, onde estão inclusos: nome da cirurgia, prótese utilizada, anestesia recebida, nível de consciência, infusões venosas, aspecto do curativo, perfusão periférica, expansibilidade torácica, presença de cateteres, sondas e drenos. A prescrição de enfermagem é feita para a fase de recuperação, e é elaborada de acordo com os problemas abordados na evolução de enfermagem. Na sala de recuperação anestésica a equipe de enfermagem presta cuidados intensivos e semi intensivos voltados para a recuperação da consciência, da estabilidade dos sinais vitais, da motilidade e da homeostase ${ }^{4}$.
Para avaliação do estado fisiológico dos pacientes, temse utilizado o índice de Aldrete-Kroulik. Este índice baseiase na avaliação dos sistemas cardiovascular, respiratório, nervoso central e muscular. Cada resposta, referente a cada item, recebe uma pontuação que varia de 0 a 2 . Após a avaliação de cada item, somam-se os pontos, obtendo-se um escore total, que subsidiará o julgamento de alta ou não do paciente da SRPA. Assim a máxima pontuação no índice é de 10 pontos e considera-se que o paciente está apto a receber alta da SRPA quando atingir pontuação igual ou superior a 8 (oito) ${ }^{10}$.

As principais complicações que podem ocorrer na SRPA são náuseas e vômitos, alteração do nível de consciência, hipoxemia, hipotensão, hipertensão e arritmias cardíacas, sendo necessária a observação constante dos parâmetros vitais e dos aparelhos conectados ao paciente, como ventilador, monitor cardíaco, oxímetro de pulso, termômetro etc ${ }^{11}$.

Todos os dados relativos à admissão e condições do paciente, devem ser anotados na ficha da SRPA, que deve ser específica para a enfermagem, sendo importante descrever na evolução de enfermagem o estado do paciente desde a sua admissão até sua alta ${ }^{11}$.

\section{Risco de Atraso no Desenvolvimento Pós Operatório}

Segundo Cianciarullo ${ }^{4}$ o período pós operatório é considerado como as primeiras 24 horas após a cirurgia, porém pode ser prorrogado por até 48 horas após o término da cirurgia para a realização da visita pósoperatória, que se inicia com a coleta de dados sobre a evolução operatória do paciente, a fim de evitar complicações contribuindo para uma recuperação adequada em um curto período de internação.

São de responsabilidade da enfermagem no pósoperatório observar o nível de consciência, principalmente em idosos, observando se o paciente está desperto, lúcido e orientado no tempo e espaço, se responde a estímulos táteis ou verbais. Após operações ortopédicas a incidência do delirium pós operatório (DPO), que ocorre com frequência em idosos, chega a ser de $50 \%$ e, especificamente, após tratamento cirúrgico de fratura de quadril nos idosos pode chegar a $62 \%$. As manifestações clínicas do DPO incluem: delirium, desorientação, dificuldade na linguagem, prejuízo no aprendizado e na 
memória. Distúrbios emocionais podem estar proeminentes, como: ansiedade, medo, irritabilidade, raiva e depressão. Ilusão e alucinação também podem estar presentes ${ }^{12}$.

Os sinais vitais devem ser monitorados durante e após a cirurgia (frequência respiratória, cardíaca, pressão arterial e temperatura); a sonda deve ser retirada 24 horas após a cirurgia ${ }^{12}$.

Para prevenir a luxação da prótese de quadril, o repouso no leito deve ser em decúbito dorsal horizontal, com membros inferiores em abdução e colocar o triângulo abdutor entre as pernas e quando em decúbito lateral, lateralizar para o lado contrário da cirurgia. O curativo da incisão cirúrgica deve estar sempre limpo e seco, em suas trocas devem-se observar manifestações de infecção: hiperemia, dor, edema e presença de exsudato. Durante a cirurgia, o paciente perde em média de 300 a $500 \mathrm{ml}$ de sangue, portanto é necessário monitorar os resultados de hemoglobina e hematócrito, avaliar perfusão periférica, monitorar sinais de TVP (dor, calor, hiperemia, edema e engurgitamento da panturrilha) e manter meias elásticas e compressor mecânico de membros inferiores ${ }^{11}$.

A dor é um sintoma comum no pós-operatório como parte do mecanismo de defesa do organismo, estando relacionada com a cirurgia propriamente dita, ao grau de traumatismo cirúrgico, ao tipo de incisão e ao posicionamento cirúrgico prolongado. Além disso, está presente em qualquer articulação com restrição de movimento normal por um período excessivo. Deverá ser observado o posicionamento para que haja alívio ou ausência da dor, sendo que, o movimento do membro afetado deve ser executado de modo seguro e coordenado, pois um músculo lesado torna-se doloroso, hipertônico e sensível. Também deverá ser observada a presença de sinais flogísticos, pois dor também é um indicativo da presença de infecção. Medidas alternativas, quando prescritas, podem minimizar a presença de edemas, hematomas e espasmos musculares, evitando assim o aparecimento do quinto sinal vital ${ }^{11}$.

Drenos de pressão negativa (Portovac) são utilizados durante o pós-operatório de ATQ para evitar o acúmulo de secreções ou a presença de hematomas bem como deiscência das suturas e uma possível infecção. Sendo assim, certos cuidados são necessários, como por exemplo: manter técnicas assépticas ao manipulá-lo certificar-se que sua extensão não esteja obstruída ou com presença de pequenos orifícios e que suas conexões estejam adequadas, evitar trações ao manipulá-lo, orientando o paciente quanto à presença deste risco e se ocasionada para que não o reintroduza, comunicado à enfermagem imediatamente e também a importância de mantê-lo abaixo do nível de inserção, sendo que a mesma deve avaliar e anotar a quantidade e aspecto do conteúdo drenado. A retirada do mesmo se faz quando apresenta secreção serosa em sua extensão ou 48 horas após a cirurgia, ou se estiver drenando menos de $30 \mathrm{ml}^{11}$.

Devido à imobilidade física deve-se avaliar integridade cutânea para prevenir úlceras por pressão e atentar para constipação devido ao uso de analgésicos opióides ${ }^{1}$.

\section{Principais Cuidados no Pós Operatório I mediato}

De acordo com o Protocolo Gerenciado Artroplastia Total do Quadril - $\mathrm{HIAE}^{1}$, a partir do pós operatório mediato, a enfermeira deve instruir, incentivar e ajudar o paciente nas mudanças de decúbito. Para sentar o paciente deve-se usar cadeira rígida com membros inferiores em abdução, adequada para a altura.

É importante, nesse período, estimular a mobilização precoce como flexão e extensão dos pés e exercícios respiratórios (inspiração profunda e expiração prolongada) e atentar para sinais de encurtamento do membro afetado, rotação inadequada e incapacidade de movimentar a extremidade e limitar a elevação da cabeceira da cama até 45 a fim de evitar a flexão aguda do quadril.

A deambulação se inicia com o andador, após autorização médica e sempre com a presença de fisioterapeuta.

\section{Complicações Pós Cirúrgicas Luxação da prótese de quadril}

É essencial a manutenção do componente da cabeça femoral no cálice acetabular. A enfermeira ensina o paciente sobre o posicionamento da perna, o que o ajuda a evitar a luxação da prótese. O uso de um triângulo abdutor é o mais indicado do que o uso de travesseiros para manter o quadril em abdução. O quadril do paciente nunca é fletido em mais de $90^{\circ}$. Para evitar a flexão do quadril, e 
nunca deve-se elevar a cabeceira do leito em mais de $60^{\circ}$. Para o uso da comadre de fratura, o paciente é orientado a flexionar o quadril não afetado e a usar o trapézio para levantar a pelve sobre a comadre. 0 paciente também é lembrado a não flexionar o quadril afetado $^{13}$.

A luxação pode ocorrer com o posicionamento que excede os limites da prótese. A enfermeira deve reconhecer a luxação da prótese. Os indicadores são os seguintes:

- Dor aumentada no sítio cirúrgico, edema e imobilização;

- Dor aguda na virilha, no quadril afetado ou desconforto aumentado;

- Encurtamento da perna;

- Rotação externa ou interna anormal;

- Capacidade restrita ou incapacidade de mover a perna;

- Sensação de "estalido" reportada no quadril.

Se uma prótese fica luxada, a enfermeira (ou o paciente quando em casa) notifica imediatamente o cirurgião, porque o quadril deve ser reduzido e estabilizado de imediato, de modo que a perna não sofra comprometimento circulatório e nervoso. Depois da redução fechada, o quadril pode ser estabilizado com a tração de Buck ou uma calha para evitar a luxação recorrente. À medida que os músculos e a cápsula articular cicatrizam, diminui a possibilidade de luxação. Os estresses sobre a nova articulação do quadril devem ser evitados durante os primeiros 3 a 6 meses $^{13}$.

\section{Trombose Venosa Profunda}

O risco de trombose venosa profunda (TVP) é particularmente grande depois da cirurgia reconstrutiva de quadril. A incidência de TVP é de $45 \%$ a $70 \%$. A ocorrência máxima é de 5 a 7 dias depois da cirurgia. Aproximadamente $20 \%$ dos pacientes desenvolvem embolia pulmonar, dos quais aproximadamente 1 a $3 \%$ dos casos são fatais ${ }^{8}$.

Em pacientes submetidos à artroplastia do quadril, ocorre embolia gordurosa (EG), que não apresenta sintomatologia grave na grande maioria dos casos, a despeito de intensas embolias, pois nesses pacientes o efeito é apenas mecânico, ou seja, a simples oclusão temporária de parte da rede capilar pulmonar. Por outro lado, se a EG for maciça a ponto de obstruir cerca de $80 \%$ da rede capilar pulmonar haverá grande elevação da pressão da artéria pulmonar e, consequente, insuficiência aguda do ventrículo direito ("cor-pulmonale" agudo), com rápida evolução para o óbito ${ }^{14}$.

\section{I nfecção}

A infecção, uma grave complicação da prótese total de quadril, pode exigir a retirada do implante. Os pacientes idosos, obesos ou desnutridos e os pacientes portadores de diabetes, artrite reumatoide, infecções concomitantes ou com grandes hematomas estão em alto risco para infecção. Como as infecções articulares totais são muito desastrosas, são empreendidos todos os esforços para minimizar sua ocorrência. As fontes potenciais de infecção são escrupulosamente evitadas. Os antibióticos profiláticos são prescritos.

Quando se utilizam sondas urinárias de demora e aparelhos de aspiração portáteis para a incisão, eles são removidos o mais breve possível, a fim de evitar a infecção ${ }^{11}$.

As infecções agudas podem ocorrer dentro de 3 meses depois da cirurgia e estão associadas às infecções superficiais progressivas ou hematomas. As infecções cirúrgicas tardias podem surgir de 4 a 24 meses depois da cirurgia e podem provocar o retorno do desconforto no quadril. As infecções que ocorrem mais de dois anos depois da cirurgia são atribuídas à disseminação da infecção através da corrente sanguínea para outro sítio no corpo. Se ocorrer infecção, são prescritos antibióticos. As infecções graves podem exigir o desbridamento cirúrgico ou a remoção da prótese ${ }^{8}$.

\section{Orientações de Alta Hospitalar}

A preocupação com a manutenção, longevidade da prótese e a capacidade dos indivíduos de atuar como pessoas produtivas fizeram com que a enfermagem desenvolvesse e aplicasse um programa educativo para autocuidado dos pacientes submetidos à ATQ. A enfermagem, ao promover a prática de autocuidado estimula o paciente a manter suas atividades de vida diária, o equilíbrio de suas funções orgânicas, seu desenvolvimento e bem estar. Mas, para que isso aconteça as orientações de alta devem ser realizadas desde que o 
paciente entra no período do pós-operatório e assim seja preparado para alta. Então, a enfermagem deve acionar os familiares, para que esses também sejam orientados quanto às orientações domiciliares com relação à terapia de autocuidado e medicamentosa.

A respeito da terapêutica de autocuidado, o paciente/cuidador dede ser orientado quanto à presença de corrimão nas estruturas da casa, principalmente no banheiro para que este não necessite de auxílio, a importância de evitar áreas com escadas, a retirada de tapetes para facilitar a locomoção e evitar quedas e movimentos de rotação. Quanto às atividades de vida diárias, deve-se fazer uso de bengalas para auxiliá-lo durante caminhadas curtas, para servir de apoio ao se levantar e para evitar sobrecarga no membro operado. Orientá-lo para que evite colchões e assentos baixos e macios a fim de prevenir uma possível flexão do quadril em grau inferior a 90 o e ausentar-se de trabalhos que exijam grandes movimentos da articulação e peso em excesso. Também se faz necessário a prática de atividades isométricas e ativas do membro operado com orientação de um profissional qualificado ${ }^{11}$.

\section{Diagnósticos e I ntervenções de Enfermagem}

Os diagnósticos de enfermagem levantados no North American Nursing Diagnosis Association (NANDA 2009-2011) $)^{15}$ aos pacientes submetidos à ATQ são: Ansiedade; Deambulação prejudicada; Risco de infecção; Integridade da pele prejudicada; Integridade tissular prejudicada; Risco de lesão por posicionamento perioperatório; Risco de disfunção neurovascular periférica; Risco de quedas; Risco de trauma vascular; Risco de desequilíbrio na temperatura corporal; Dor aguda; Déficit no autocuidado; Risco de sangramento.

Dentre os diagnósticos levantados, destacam-se principalmente:

\section{Ansiedade}

Definição: Vago e incômodo sentimento de desconforto ou teor acompanhado por resposta autonômica (a fonte é frequentemente não específica ou desconhecida para o indivíduo), sentimento de apreensão causada pela antecipação de perigo. É um sinal iminente e permite ao indivíduo tomar medidas para lidar com a ameaça.

Características definidoras: Aflito; ansioso; apreensivo; irrequieto; nervoso; preocupado; receoso; medo de consequências inesperadas; agitação; aumento da tensão; aumento da pressão sanguínea.

Fatores de risco: Estresse; mudança no estado de saúde. Resultados esperados: Minimizar ansiedade a um nível controlado.

\section{I ntervenções}

1. Explicar ao paciente detalhadamente as técnicas e procedimentos a serem feitos, além de orientá-lo quanto aos cuidados pós-operatório. Se necessário pedir que ele repita tais orientações;

2. Estimular o paciente a verbalizar suas preocupações quanto ao procedimento cirúrgico proporcionar informações precisas e de maneira simplificada que abone suas dúvidas, passando-Ihe confiança e segurança;

3. Oferecer medidas de conforto e ambiente calmo.

\section{Risco de I nfecção}

Definição: O estado em que um indivíduo corre grande risco de ser invadido por organismos patogênicos.

Fatores de risco: Defesas primárias e secundárias inadequadas; procedimentos invasivos e trauma.

Resultados esperados: Diminuir risco de infecção para que não haja complicações perioperatórias.

\section{Intervenções:}

1. Providenciar cuidado adequado à pele em áreas edemaciadas;

2. Observar sinais flogísticos na incisão cirúrgica e dreno;

3. Utilizar técnicas assépticas e estéreis em procedimentos invasivos:

4. Administrar/monitorar antibióticos e imunização como profilaxia, conforme prescrição médica;

5. Monitorar quanto à elevação da temperatura a cada 06 horas.

\section{Dor aguda}

Definição: experiência sensorial e emocional desagradável que surge de lesão tissular real ou potencial ou descrita em termos de tal lesão início súbito ou lento, de intensidade leve a intensa, constante ou recorrente, sem 
um término antecipado ou previsível e com uma duração de mais de 6 meses.

Fatores relacionados: Agentes lesivos.

Características definidoras: Comunicação verbal de dor; comportamento distraído; expressão facial; respostas autônomas (pressão arterial - PA, pulso - $P$, frequência respiratória - FR, frequência cardíaca - FC); comportamento protetor.

Resultados esperados: controlar a dor; Alivio a dor

\section{I ntervenções}

1. Realizar uma avaliação abrangente da dor (duração, local, gravidade, frequência);

2. Fazer avaliação da dor cada vez que ela aparecer (escala);

3. Oferecer medidas de conforto (mudança de posição; uso de calor/frio);

4. Administrar analgésicos conforme prescrição médica, avaliar a resposta da medicação ao alivio da dor;

5. Aliviar a dor para que haja uma melhora na qualidade de vida.

\section{Conclusão}

A enfermagem tem um papel fundamental no processo perioperatório dos pacientes submetidos à ATQ. A SAE é uma ferramenta que auxilia o enfermeiro a prestar um cuidado individualizado e amplo abrangendo as orientações em todas as fases da internação e também no domicilio, visando eliminar a possibilidade de iatrogenias e proporcionar uma reabilitação adequada e ainda capacitar o cuidador para alta hospitalar.

\section{Referências}

1. Hospital Israelita Albert Einstein. Diretrizes assistenciais - protocolo gerenciado artroplastia total do quadril. Versão Eletrônica Atualizada. 2009.

2. Duarte LTD, et al. Anestesia peridural lombar ou bloqueio do plexo lombar combinados à anestesia geral: eficácia e efeitos hemodinâmicos na artroplastia total do quadril. Revista Brasileira de Anestesiologia. 2009; 59(6).
3. Takata ET. Artroplastia total e parcial do quadril (aula multimídia). São Paulo: UNIFESP. 1999. Disponível em: <http://learning.einstein.br>. Acesso em: 01 mar 2012.

4. Cianciarullo $\mathrm{TI}$, et. al. Sistema de Assistência de Enfermagem: evolução e tendências. São Paulo: Ícone. 2008.

5. Tamegushi AS, et al. Capacidade funcional de idosos com osteoartrite de joelhos e quadril. Londrina: Revista Espaço para a Saúde. 2008; 9(2):08-16. Disponível em: <www.ccs.uel.br/espcoparasaude>. Acesso em: $14 \mathrm{mar}$ 2012.

6. Eliopoulos C. Enfermagem gerontológica. Porto Alegre: Artmed. 2005.

7. Meeker MH, Rothrock JCA. Cuidados de enfermagem ao paciente cirúrgico. Rio de Janeiro: Guanabara Koogan. 2008.

8. Smeltzer SC, Bare BG. Brunner \& Suddart: Tratado de enfermagem médico cirúrgica. Rio de Janeiro: Guanabara Koogan. 2002.

9. Cavalcanti IL, Cantinho FAF, Assad A. Medicina perioperatória. Rio de J aneiro: Sociedade de Anestesiologia do Estado do Rio de Janeiro. 2006; 105:932-934.

10. Carvalho R, Bianchi ERF. Enfermagem em centro cirúrgico e recuperação. São Paulo: Manole. 2007.

11. Tashiro MTO. Assistência de enfermagem em ortopedia e traumatologia. São Paulo: Atheneu. 2001.

12. Barbosa FT, Cunha RM, Pinto ALCLT. Delirium pósoperatório em idosos. Campinas: Revista Brasileira de Anestesiologia. 2008; 58(6): 665-670.

13. Smeltzer SC, Bare BG. Brunner \& Suddart: Tratado de enfermagem médico cirúrgica. Rio de Janeiro: Guanabara Koogan. 2005.

14. Filomento LTB, Carelli CR, Silva NCLF, Barros Filho TEP, Amatuzzi MM. Embolia gordurosa: uma revisão para a prática ortopédica atual. São Paulo: Acta Ortopédica Brasileira. 2005; 13(4):196-208.

15. North American Nursing Diagnosis Association NANDA. Diagnósticos de Enfermagem da NANDA: definições e classificações 2009-2011. Porto Alegre: Artmed. 2010. 